\title{
A SIMPLE INTERVAL-PROGRAMMING CIRCUIT
}

\author{
Fogle C. Clark and Carter W. Caudill
}

UNIVERSITY OF KENTUCKY

A reinforcement lock-up unit for use with interval schedules is described. The circuit is designed to be used with Gerbrands punched-tape programmers or with cam timers. The unit can be inexpensively constructed, yet provides the same features as commercially available units. It is easily used in simple FI's or VI's, in "limited-hold" schedules, in multiple or conjunctive schedules, or as a self-recycling timer.

A circuit diagram is presented in Fig. 1. The unit is constructed on a snap-on panel for attachment to 24-volt D. C. power rods. The A. C. line current for the timer motor is brought in separately through a plug, and may be externally switched. External connections to the timer motor and switch are also made through a Jones plug and socket. All 24-volt D.C. switching terminals are brought out to $\mathrm{Nu}$-Way connector studs on the front panel. If inexpensive relays are used (e.g., Relay A, Potter and Brumfield Type KA11D, and Relay B, Potter and Brumfield Type MH17D), the cost of parts for the unit will run about $\$ 15.00$; with telephone types (e.g., Clare Type J), costs will be $\$ 5.00$ to $\$ 10.00$ more.

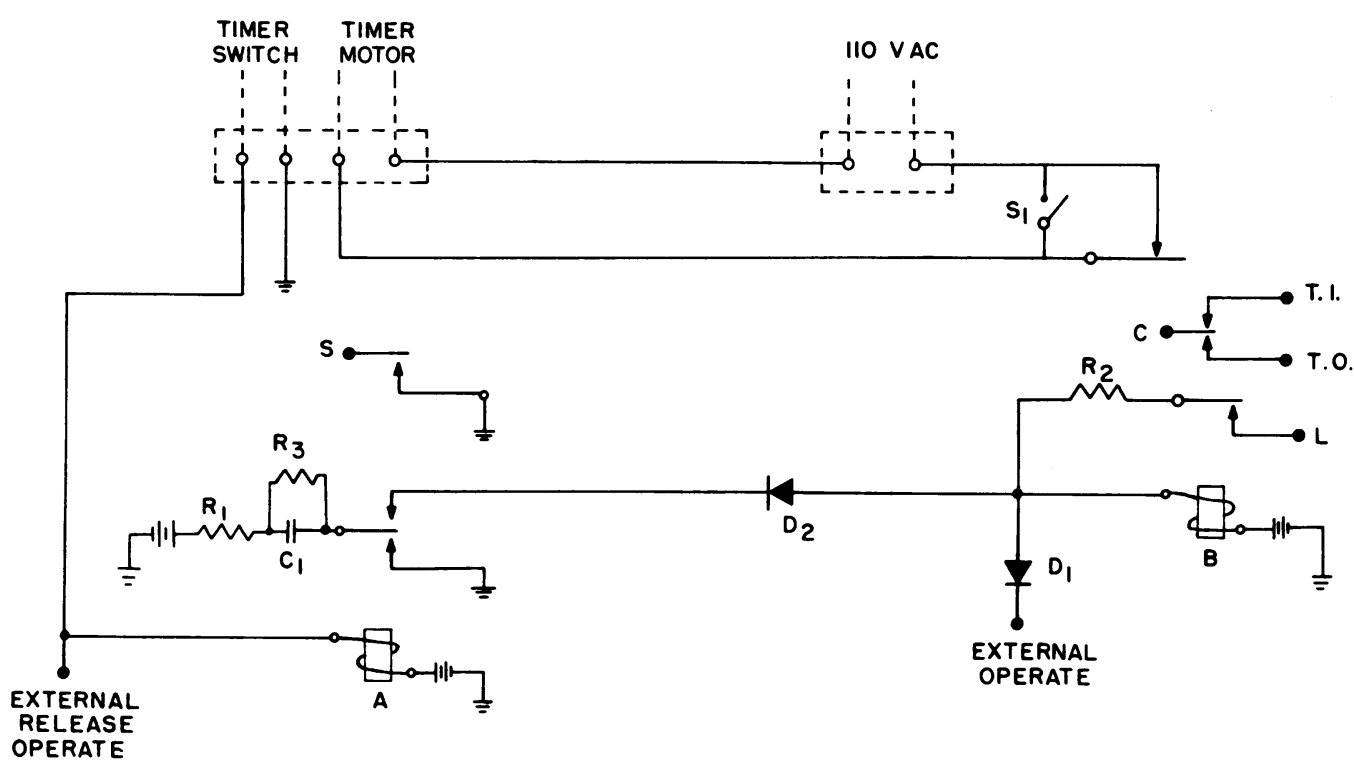

Figure 1 .

For use with Gerbrands punched-tape timers in programming Fl's or VI's, the unit is connected as follows: timer switch and motor, and also A. C. line, are plugged in; response pulses are connected to C and the "time-out" (T.O.) side of the transfer contacts on Relay B to the external reinforcement-control relay; external ground is supplied to Stud L from the normally closed side of a transfer contact on the reinforcement relay; and finally, $S_{1}$ is open.

'This equipment was developed under a grant to the first author from the Faculty Research Fund, University of Kentucky. 
With these connections made, the timer runs so long as Relay $B$ is unoperated. When the timer switch enters a hole in the tape, Relay A is operated, charging Condenser $\mathrm{C}_{1}$ through $R_{1}$. Simultaneously, a ground signal appears on Stud $S$. As the timer switch emerges from a hole in the tape, ground is removed from Stud $\mathrm{S}$ and Condenser $\mathrm{C}$ discharges, operating Relay B. Relay B locks up on the ground supplied to Stud L from the reinforcement relay ( $R_{2}$ limits the current through Relay B during lock-up). When Relay $B$ locks up, the timer is halted and a response pulse will pass through the T.O. side of its transfer contacts to the reinforcement relay. When a reinforcement occurs, the ground is removed from $\mathrm{L}$, unlocking Relay $\mathrm{B}$ which restarts the timer and prevents the occurrence of further reinforcements until the next cycle.

When it is desired to continue timing during lock-up, $S_{1}$ is closed so that each interval is shortened by the amount of the previous lock-up. Additional reinforcements may be programmed into the schedule from an external device by means of the "external operate" or "external release operate" studs. The former will lock up Relay B directly on the "make" of an external ground, the latter indirectly on the "break" of an external ground. Where a schedule with "limited hold" is desired, an external timer switch which opens at the end of the hold time may be inserted between Stud $L$ and the ground from the reinforcement relay. (This timer must be reset by reinforcements.) The unit may be used as a continuously recycling timer by simply removing the locking ground from Stud L. If ground is then connected to $\mathrm{C}$ studs on the transfer switches of Relay $\mathrm{B}$, a brief pulse will be emitted on the T.O. studs at the end of each cycle. (For recycling operation over long periods of time, $S_{1}$ should be closed in order to avoid the cumulation of small timing errors due to the break in the A. C. circuit through Relay B.) To use the unit in a multiple schedule, all connections are made as for FI-VI operation, and the 110-volt A. C. supply is externally switched by the schedule-selecting switch. Under all modes of operation involving the activation of Relay $A$, the $S$ stud may be used to provide ground signals preceding the operation of Relay B. These signals may be used to control a discriminative stimulus, the duration of which is determined by the length of hole punched in the timer tape.

The only critical values in the circuit of Fig. 1 have to do with the operate and release times of Relay B. Then, $C_{1}$ must be large enough to operate Relay B reliably on the break of a brief ground pulse to the "external release operate" stud. The values of $C_{1}$ and $R_{1}$ will be in the range 50-100 microfarads and 100-300 ohms, respectively. Resistor $R_{1}$ serves merely to limit the charging current through $C_{1}$ when Relay $A$ is operated. A simple procedure for adjusting these values is the following. Pick trial values of $C_{1}$ and $R_{1}$, say 50 microfarads and $100 \mathrm{ohms}$; then, operate Relay A with a brief pulse on the "external release operate" stud. This pulse determines the charging time of $C_{1}$, and should be much shorter than that normally delivered by the timer switch. Use of a 30-40-millisecond pulse delivered by a response-pulse shortener (Gollub, 1958) will insure reliable activation from programming units other than the timer. If Relay B fails to operate reliably on the break of a brief pulse to Relay $A$, the trial value of $C_{1}$ must be increased. When a large-enough value of $C_{1}$ is found, the value of $R_{1}$ should be increased gradually until Relay $B$ occasionally fails to operate. About $50 \%$ of this value will usually be found satisfactory for $R_{1}$, provided that this resistance is at least three or four times the supply voltage, and provided Relay B remains operated for at least 30-40 milliseconds when no ground is supplied to Stud L. Either of these conditions failing, the value of $C_{1}$ should be increased and the procedure repeated. After these adjustments are made, the unit should be locked up 
to the reinforcement relay and tested to determine if a response pulse passed through the T. O. side of the transfer contacts on Relay B allows the reinforcement relay to remain operated long enough to release Relay B. A break time of 30-40 milliseconds on the locking ground to Stud L should be sufficient to insure release of Relay B. (If necessary, the value of $R_{2}$ may be increased slightly; this is normally set to hold Relay $B$ about halfway between pull-in and drop-put currents.)

The rectifier $D_{2}$ in the discharging circuit from $C_{1}$, together with the by-pass resistor $R_{3}$, serves to prevent back-charging of $C_{1}$ from the ground on Stud $L$ when Relay $B$ is locked up. ${ }^{2}$ Texas Instruments IN2071 will serve for $D_{2}$, with $R_{3}$ at about 5000 ohms. (Resistor $R_{3}$ must be small with respect to the reverse resistance of $D_{2}$, yet large enough not to interfere with the discharge of $C_{1}$ through Relay B.) Adjustment of $C_{1}$ and $R_{1}$ should be accomplished with $D_{2}$ and $R_{3}$ in the circuit, and with the power supply under a normal programming load.

Spark suppression is not shown in Fig. 1. Assuming other relays in the system are suppressed, contact protectors (e.g., Federal Type 8A2PS1) placed across the relay coils will be satisfactory. The rectifier $D_{1}$ in the "external operate" path to Relay B serves to prevent $C_{1}$ from discharging through other relays and also prevents ground from appearing on the "external operate" stud when Relay B is locked up. Inexpensive selenium rectifiers are adequate for these purposes.

\section{REFERENCE}

Gollub, L. R. A simple pulse shaper, J. exp. anal. Behav., 1958, 1, 122.

Received July 24, 1959.

\footnotetext{
${ }^{2}$ Inclusion of $D_{2}$ and $R_{3}$ in this circuit was suggested by Dr. D. G. Anger, and inexpensively eliminates the troublesome problem of back-charging $C_{1}$ through the locking path on Relay $B$. This method will not work satisfactorily with selenium or other rectifiers having low reverse resistance. (With a low reverse resistance on $D_{2}$ a charge will trickle onto $C_{1}$ from Stud $L$, and introduce variations in the break required on Stud $L$ to allow release of Relay B. Such variations will be a function of the duration of the previous lock-up.) Another, somewhat less satisfactory, method of solving this problem is to eliminate $D_{2}$ and $R_{3}$ from the circuit entirely. Then, $C_{1}$ will be recharged through $R_{3}$ whenever Relay $B$ is locked up, and will hold Relay $B$ momentarily as it discharges when the locking ground is removed from Stud L. This will usually necessitate modification of the reinforcement-control circuit to insure that the reinforcement relay remains operated long enough to allow $\mathrm{Re}$ lay $B$ to release. This can be accomplished by inserting a condenser and resistor in series between the operate terminal of the reinforcement relay and the "plus" side of the D. C. supply.
} 